\title{
Adaptive OFDM system for communications over the indoor wireless optical channel
}

\author{
O. González, R. Pérez-Jiménez, S. Rodríguez, J. Rabadán and A. Ayala
}

\begin{abstract}
The authors propose an adaptive orthogonal frequency division multiplexing (OFDM) system for communications over the indoor wireless diffuse optical channel. This channel can be characterised as short-term stationary, with severe attenuation and multipath-induced penalty, and high dependence on the space distribution of emitters and receivers. We have chosen OFDM systems because of their capability of supporting high data rates without channel equalisation. They also mitigate the quality of service fluctuations induced when space distribution of emitters and receivers varies. The performance of the new proposed scheme is compared with that of an adaptive system described in a previous work. The obtained results show that a significant increase of the system throughput is attained over noisy wireless optical channels.
\end{abstract}

\section{Introduction}

The emergence of indoor wireless local area networks and portable information terminals offer flexible and economical alternatives to wired networks. For wireless transmission, the infrared (IR) medium is an attractive alternative to radio in several environments, as it possesses an enormous amount of unregulated bandwidth and because of the absence of interference between links operating in adjacent rooms [1]. However, it presents drawbacks as severe attenuation and intersymbol interference (ISI) due to multipath propagation, that degrade the quality of the transmission. Equalisation techniques have been used in single-carrier modulation schemes so as to mitigate multipath-induced distortion, however, they increase excessively the receiver complexity for high data rates [2, 3]. Spread spectrum techniques have also been extensively studied, but they reduce severely the bandwidth efficiency $[4,5]$. Multiple-subcarrier modulation has been also proposed $[6,7]$. It is more bandwidth efficient than singlecarrier modulation schemes and it experiences little distortion. It also allows multiple simultaneous use of the channel through allocation of the several subcarriers for each individual user. A particularly attractive technique is orthogonal frequency division multiplexing (OFDM), which uses multiple carriers overlapped in the frequency domain $[8,9]$. OFDM systems are able to support high data rates without needing channel equalisers, as the aggregate throughput is distributed over the set of subcarriers. In contrast, the quality fluctuations of time-varying wireless channels can

\footnotetext{
(C) The Institution of Engineering and Technology 2006

IEE Proceedings online no. 20050081

doi:10.1049/ip-opt:20050081

Paper first received 7th September and in revised form 13th December 2005

O. González, S. Rodríguez, and S. Ayala are with the Department of Fundamental and Experimental Physics, Electronics and Systems, University of La Laguna, La Laguna 38203, Tenerife (Canary Islands), Spain

R. Pérez-Jiménez and J. Rabadán are with the Department of Signals and Communications, University of Las Palmas de Gran Canaria, Las Palmas de Gran Canaria (Canary Islands) 35017, Spain

E-mail: oghdez@ull.es
}

be mitigated with the aid of frequency-domain channel equalisation. In addition, an appropriate modulation mode can be adaptively allocated to each subcarrier according to sub-band quality, increasing the aggregate throughput. Finally, OFDM modems use the discrete Fourier transform to replace the function of the banks of the modulators/ demodulators of the sub-channels, thus significantly reducing the implementation complexity.

\section{Optical channel model}

There are several models used to estimate the impulse response of IR wireless indoor channel. We have selected a Monte Carlo ray-tracing algorithm [10-12]. In the algorithm, every ray is generated at the emitter position with a probability distribution equal to its radiation pattern. The power of each generated ray is initially the source power $(1 \mathrm{~W})$ divided by the number of rays used to discretise the source. When a ray impinges on a surface, the reflection point is converted into a new optical source, thus a new ray is generated with a probability distribution provided by the reflection pattern of that surface. The process continues during the simulation time. After each reflection, the power of the ray is reduced by the reflection coefficient $(\rho)$ of the surface and the reflected power which reaches the receiver ( $p_{i, k}, i$ th ray, $k$ th time interval) is computed. The Phong's model was used to describe the reflection pattern of surfaces [13]. This model is able to approximate the behaviour of those surfaces that present a strong specular component: it takes into account the reflection pattern as the sum of both diffuse and specular components. Therefore surface characteristics are defined by two new parameters: the percentage of incident signal that is reflected diffusely $r_{\mathrm{d}}$ and the directivity of the specular component of the reflection $m$. The total received power in the $k$ th time interval (width $\Delta t$ ) is computed as the sum of the power of the $N_{k}$ rays that contribute in that interval.

$$
p_{k}=\sum_{i=1}^{N_{k}} p_{i, k}
$$


As we have adopted a receiver responsivity of $1 \mathrm{~A} / \mathrm{W}$, the channel impulse response is given by

$$
h(t)=\sum_{k=0}^{K-1} p_{k} \delta(t-k \Delta t)
$$

where $K=t_{\max } / \Delta t$, and we have assumed as the time origin the arrival of the line-of-sight (LOS) component. The assumption of a receiver responsivity of $R=1 \mathrm{~A} / \mathrm{W}$ and an active area of $A=1 \mathrm{~cm}^{2}$ allows us to obtain normalised values of the impulse responses $[1,7,12,13]$, but the use of more real optical detectors' values only yields a proportional change on the received optical power. Therefore the results presented in this work are directly extensible to any other receiver characteristics.

\section{Optical adaptive OFDM system model}

The block diagram of the proposed wireless optical OFDM system is shown in Fig. 1. First, $\Sigma_{p} b_{p}$ data bits are generated and modulated by $P 2^{b_{p}}$-QAM modulators ( $P$, carriers; $b_{p}$, the number of bits conveyed by carrier $p$ ). Then, $N-P$ zeros (one of them before, corresponding to the DC value) are padded to the $P$ symbols generated by the modulators, and the complex conjugate of the mirror of the word of $N$ symbols is added to the latter before computing inverse fast Fourier transform (IFFT). The outgoing IFFT symbol is a real sequence of $2 N$ points and it can drive directly the light source after adding an appropriate DC offset to avoid lower clipping. Moreover, a cyclic prefix $(L-2 N$ samples extension) is inserted to combat ISI. The symbol duration is $T_{\mathrm{s}}=(1 / \Delta f) \mathrm{s}$ (with the carriers $\Delta f$ hertz far apart), and in this time $L$ samples are transmitted. This transmitted signal is distorted by channel response (channel plus transmitter low-pass and receiver antialiasing filters) and corrupted by noise (channel noise, preamplifier thermal noise and photodiode shot noise). The overall noise process is modelled as zero-mean additive white Gaussian noise (AWGN) with two-sided power spectral density $N_{0} / 2$. We assume that the receiver is synchronised to the LOS signal. Once the corrupted cyclic prefix is removed, the fast Fourier transform of $2 N$ points is computed by the receiver. Only the first $P$ points (after DC value) are considered and a channel equalisation is performed over them. It is a very simple operation. A known training sequence (TS) $\left(X_{p}, p=1, \ldots, P\right)$ is transmitted because of the slow time-varying nature of wireless optical channels, and the channel response of each sub-band $\left(H_{p}\right)$ can be estimated from the $P$ received symbols $\left(Y_{p}\right)$ as follows

$$
\hat{H}_{p}=Y_{p} / X_{p}
$$

These values can be used to outweigh the distorting channel effects before symbol demodulation. Note that $\hat{\mathrm{H}}$ values are distorted by noise and therefore several TS can be transmitted and then we can use the mean values of the estimates for a better channel characterisation.

Because of the slow time-varying nature of wireless optical channels, the use of several TS does not represent a meaningful amount of transmission overhead. In fact, this task is run between periods of time where the transmission of a huge amount of OFDM symbols has been completed. At the velocity of a person walking, several thousands of OFDM symbols (even millions in some cases) can be transmitted without any significant change in the channel response. Also, a good knowledge of the channel response can help to make a better use of the channel capacity by means of an adaptive subcarrier modulation system. In a wireless optical channel, different subcarriers will undergo different gains. Therefore the modulation mode of each transmission subcarrier, that is the number of transmitted bits assigned to each subcarrier, can be adaptively tailored to the channel characteristics. The receiver system has to estimate the instantaneous channel response and then it has to determine the most suitable modulation mode of each transmission subcarrier. It is clear that there is an additional amount of transmission overhead, as the receiver system has to inform the transmitter about the number of bits assigned to each subcarrier. However, as we have explained previously this does not really represent a problem in an indoor low mobility environment.

The effective demodulation signal-to-noise ratio (SNR) can be computed at the receiver as follows (Fig. 1). After each OFDM symbol demodulation, the retrieved data bits are modulated again and the average SNR of received QAM symbols is computed, using outgoing QAM modulators symbols as reference (therefore we are assuming error-free transmission). The calculation of the effective demodulation SNR is given by

$$
\overline{\mathrm{SNR}}_{\mathrm{eff}}(\mathrm{dB})=10 \log _{10} \frac{\overline{\left|s_{i}\right|^{2}}}{\overline{\left|r_{i}-s_{i}\right|^{2}}}
$$

where $s_{i}$ and $r_{i}$ are the transmitted and received (before demodulation) data symbols, respectively. From (4), it can

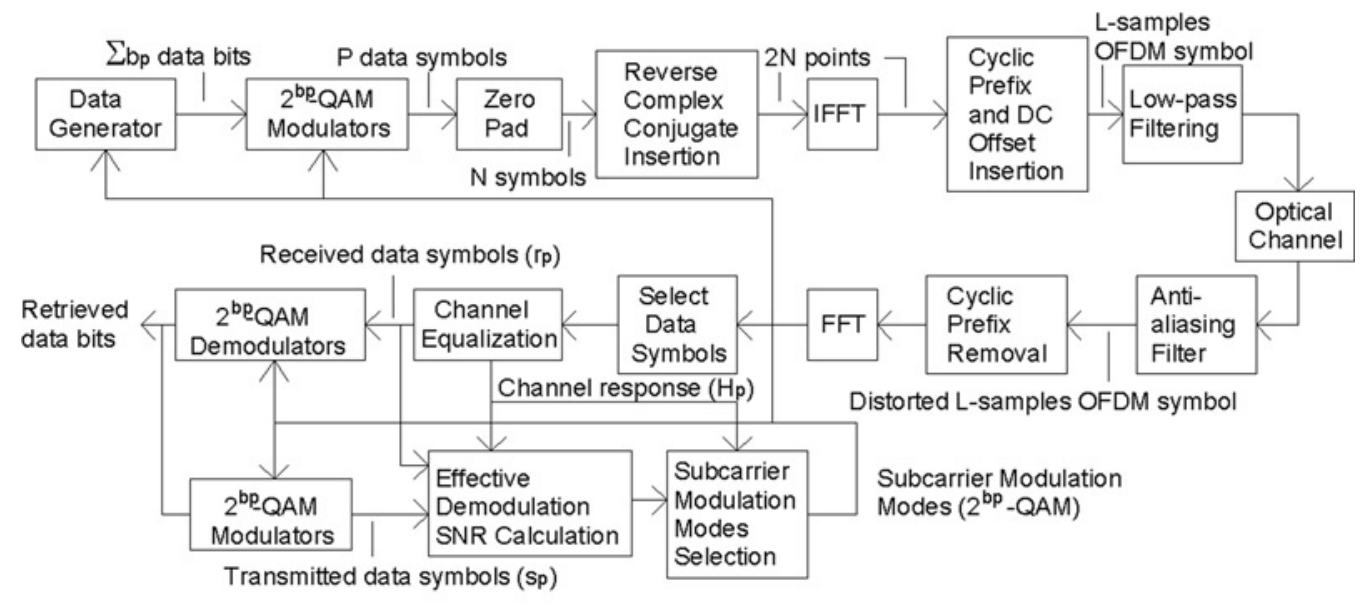

Fig. 1 Adaptive OFDM system for wireless optical indoor channels 


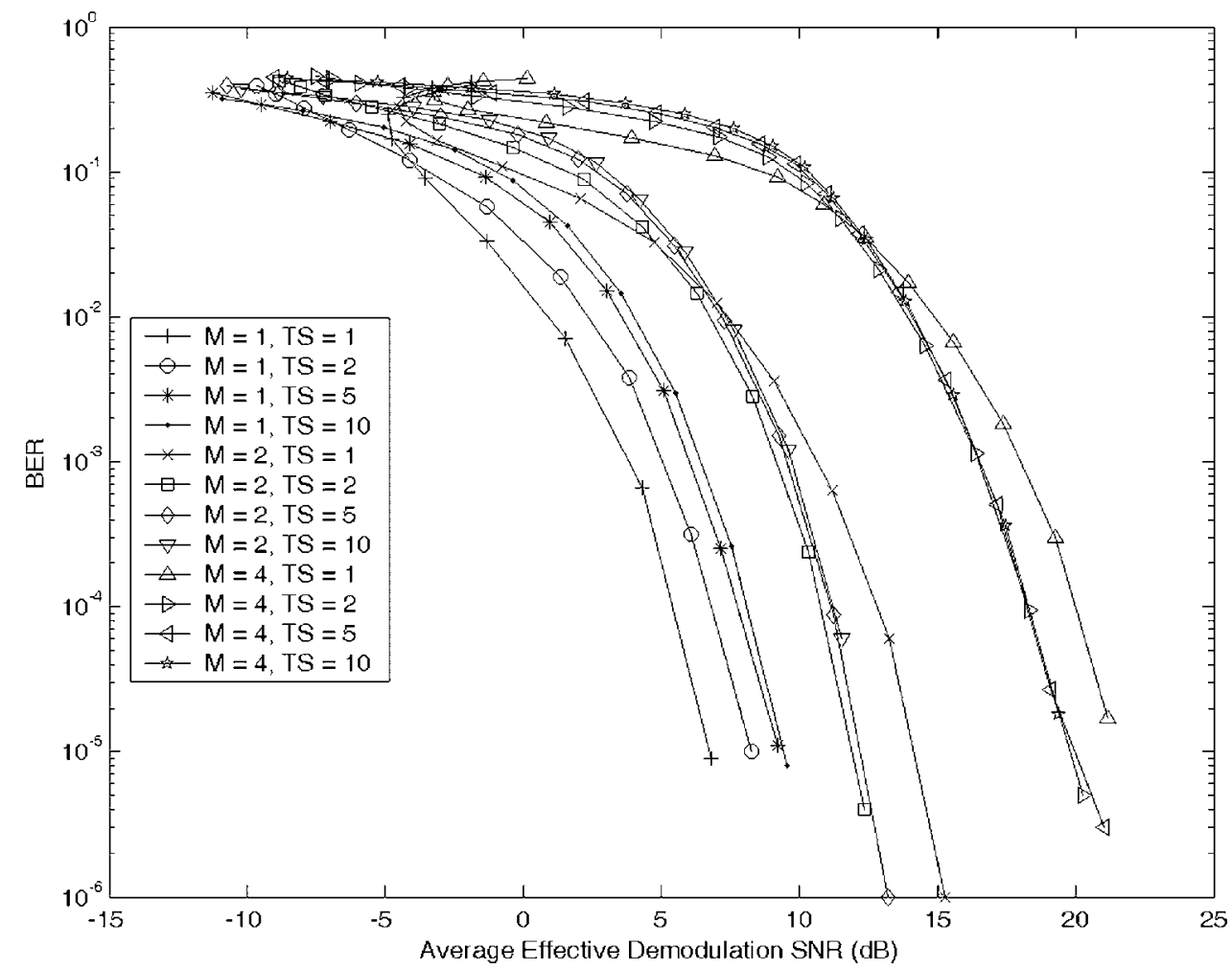

Fig. 2 BER of $2^{M}$-QAM modulated OFDM against the average effective demodulation SNR over AWGN channel for different number of TS

be deduced that the effective SNR is an average over all constituent data symbols of each received OFDM frame.

The bit error rate (BER) curves of each constituent $2^{M}$-QAM modulation mode can be used to find the SNR values that satisfies a specific target BER requirement [14]. So, using a simple extrapolation of the BER curves against the effective demodulation SNR obtained over AWGN channel (Fig. 2), we can determine the required SNR values for several BER thresholds and number of TS used. These values can be used as switching levels for a subcarrier-by-subcarrier adaptive OFDM system [15]. The effective demodulation SNR is not the actual channel SNR value, but an estimation obtained at the receiver. For this reason, the estimation can be erroneous when the channel SNR is very low and the channel response estimation is not good. This is the case when only a TS $=1$ is used to estimate the channel response. Thus, at low channel SNR values lower effective demodulation SNR's can be observed and the BER curves can even experiment increases when the channel SNR decreases. This can lead to a malfunction of the system and then a larger number of TS for estimating the channel response (e.g. TS $=10$ ) is preferred. The channel response estimation and the mean effective demodulator SNR of each received OFDM symbol can be used to determine each sub-band SNR as follows

$$
\mathrm{SNR}_{p}=\left(\frac{\left|\hat{H}_{p}\right|^{2}}{\overline{\left|\hat{H}_{p}\right|^{2}}}\right) \overline{\mathrm{SNR}}_{\mathrm{eff}}
$$

where $\overline{\left|\hat{H}_{p}\right|^{2}}$ represents the average over the estimated magnitude response. In the previous system, the sub-band SNR value can be compared with switching levels for picking out the modulation mode (including 'no transmission' i.e. $b_{p}=0$ ) that ensures the instantaneous BER always remains below a certain threshold [15]. In addition, disabled subcarriers owing to low SNR values must carry on sending dummy data in order to compute their current sub-band SNR by means of (5). These dummy symbols can be known by receiver in order to avoid an erroneous calculation of the effective demodulation SNR.

A further improvement can be carried out if subcarriers with higher SNR values between two switching levels are prompted to use the next modulation mode, whenever the average error probability do not overcome the imposed threshold. Let be $b_{p}$ be the number of bits conveyed by subcarrier $p$, and $P_{p}$ the bit error probability of the subcarrier when the modulation mode $2^{b_{p_{-}} \mathrm{QAM}}$ is used, the average error probability is given by

$$
P_{\mathrm{avg}}=\frac{1}{B} \sum_{p=1}^{P-1} b_{p} P_{p}\left(b_{p}, \mathrm{SNR}_{p}\right)
$$

where $B$ is the total throughput of the system

$$
B=\sum_{p=0}^{P-1} b_{p}
$$

The bit error probability $P_{p}\left(b_{p}, \mathrm{SNR}_{p}\right)$ can be determined from the estimated SNR of each sub-band $\left(\mathrm{SNR}_{p}\right)$ given by (5) and the BER curves of Fig. 2. Initially, the modulation modes of subcarriers are set up to those that overcome the switching levels for a certain target BER, as in the previous system. However, in this new system, later the modulation modes are successively increased for the 'best subcarriers' trying to augment $B$, while ensuring that $P_{\text {avg }}$ do not overcome the imposed BER threshold value. With the aim of maximising the total throughput $B$, while the average error probability $P_{\text {avg }}$ is maintained below the imposed maximum BER, we have adopted as criterion for selecting the 'best subcarriers' those that present a minimum increase in the next expression

$$
\theta_{p, \Delta B}=\frac{b_{p, \Delta B} P_{p, \Delta B}-b_{p} P_{p}}{b_{p, \Delta B}-b_{p}}
$$

where the values indicated with the subindex $\Delta B$ are referred to the new values of the parameters $\left(b_{p}\right.$ and 
$\left.P_{p}\left(b_{p}, \mathrm{SNR}_{p}\right)\right)$ when the modulation mode of subcarrier $p$ is increased to the next constituent modulation mode. When the 'best subcarrier' is selected by means of (8), the new average error probability given by (6) is re-calculated using the values referred to the new modulation mode and it is verified that the target BER is not overcome. If the previous condition is fulfilled, new 'best subcarriers' are looked for subsequently until the target BER is overcome. The selection criterion given by (8) is not optimum, but it allows to select with a high probability the 'best subcarriers' that maximising the total throughput with a minimum cost in the average error probability. Also, it is a very simple and efficient method that can be used in practical applications. The results observed with this new scheme show a near optimum behaviour of the system and a significant enhancement of its performance with respect to the previous one described in Gonzalez et al. [15].

\section{Simulation results}

The IR channel configuration used in the study of the proposed system model is a furnished laboratory. The main characteristics of this laboratory are described in Table 1 . The room has a wide variety of reflecting materials, including wood, glass (windows), cement and ceramic floor. The height of each piece of furniture is $1.60 \mathrm{~m}$. The length of each one is $4.60 \mathrm{~m}$ and the width is $1.55 \mathrm{~m}$. The receivers are placed $0.75 \mathrm{~m}$ above the floor and we assume that they are moving along the room. The pieces of furniture are distributed along the width of the room, and each one is separated by $1.75 \mathrm{~m}$ from each other. The emitter and receivers were considered pointing straight up. The emitter was placed at the center of the laboratory on top of the highest piece of furniture level, where contact with the ceiling was unobstructed. The emitter is considered to have a Lambertian radiation pattern with half-power beamwidth (HPBW) $=45^{\circ}$. This forms a nondirected LOS link. The time-varying channel frequency response during receiver movement was determined from the impulse responses obtained using a Monte Carlo raytracing algorithm [12] for the different positions of the

Table 1: Parameters for simulation

\begin{tabular}{|c|c|c|c|}
\hline Laboratory & Emitter & Receiver & \\
\hline Length $(x): 5.80 \mathrm{~m}$ & Mode: 2 & Area: $1 \mathrm{~cm}^{2}$ & \\
\hline Width $(y): 13.25 \mathrm{~m}$ & $\begin{array}{l}\text { Position: } \\
\qquad(2.9,6.6,1.6)\end{array}$ & FOV: $70^{\circ}$ & \\
\hline \multirow[t]{4}{*}{ Height (z): $3 \mathrm{~m}$} & Elevation: $0^{\circ}$ & Responsivity: $1 \mathrm{~A} / \mathrm{W}$ & \\
\hline & Azimuth: $0^{\circ}$ & Height (z): $0.75 \mathrm{~m}$ & \\
\hline & & Elevation: $0^{\circ}$ & \\
\hline & & Azimuth: $0^{\circ}$ & \\
\hline Materials & $\rho$ & $r_{\mathrm{d}}$ & $m$ \\
\hline Ceramic floor & 0.16 & 1 & - \\
\hline Cement & 0.57 & 1 & - \\
\hline Wood & 0.73 & 1 & - \\
\hline Varnished wood & 0.75 & 0.3 & 97 \\
\hline Glass & 0.03 & 0 & 280 \\
\hline
\end{tabular}

Resolution $(\Delta t): 0.2 \mathrm{~ns}$

Simulation time $\left(t_{\max }\right): 120 \mathrm{~ns}$

Number of rays $\left(N_{\mathrm{R}}\right): 100000$ receiver (see Table 1 for simulation parameters). In Fig. 3, the channel response for several receiver locations is displayed. Notice that we have represented the electrical power, not the received optical power. The average received optical power is always bigger than $-40 \mathrm{dBm}$, even at the room corners. A more realistic value of detector's physical area and responsivity (e.g. $A=0.25 \mathrm{~cm}^{2}$ and $R=0.5 \mathrm{~A}$ / W) lead to a loss of received power (about $9 \mathrm{~dB}$ for this example i.e. we have a minimum of $-49 \mathrm{dBm}$ of received optical power) with respect to the assumed normalised values $\left(A=1 \mathrm{~cm}^{2}\right.$ and $\left.R=1 \mathrm{~A} / \mathrm{W}\right)$. Nowadays, there are commercial detectors that can perform adequately with this power levels. On the other hand, all of the subsequent results are represented as a function of the current SNR and the absolute values of the received optical power and noise are not really relevant for the results interpretation. Moreover, emitter and receiver configurations can be modified to obtain acceptable SNR values at the receiver in order to achieve a desired system performance, for example, using receivers with optical concentrators or lenses, multibeam emitters, avalanche phototides (APDs), and so on.

Several simulations were performed over previous slowly time-varying optical channel, assuming a receiver speed of $2 \mathrm{~m} / \mathrm{s}$. The symbol rate was $1 \mathrm{Msymbol} / \mathrm{s}$. A cyclic prefix extension of 32 samples was used, which leads to a transmission rate of 160 Msamples/s. The transmitted OFDM signal bandwidth is lower than $80 \mathrm{MHz}$ and the cut-off frequency of used low-pass filters takes this value. Using
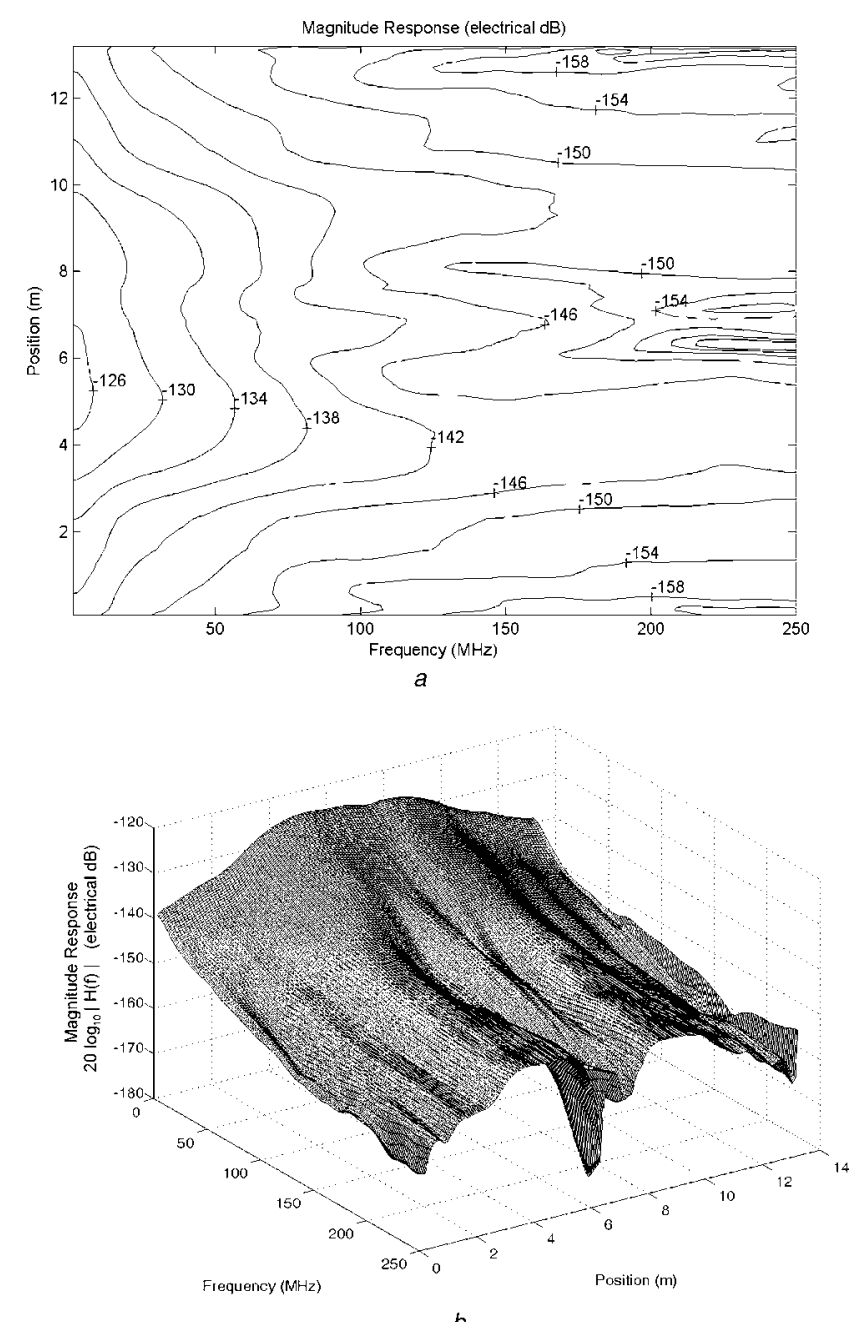

Fig. 3 Time-varying channel frequency response (contour map and $3 D$ representation) 


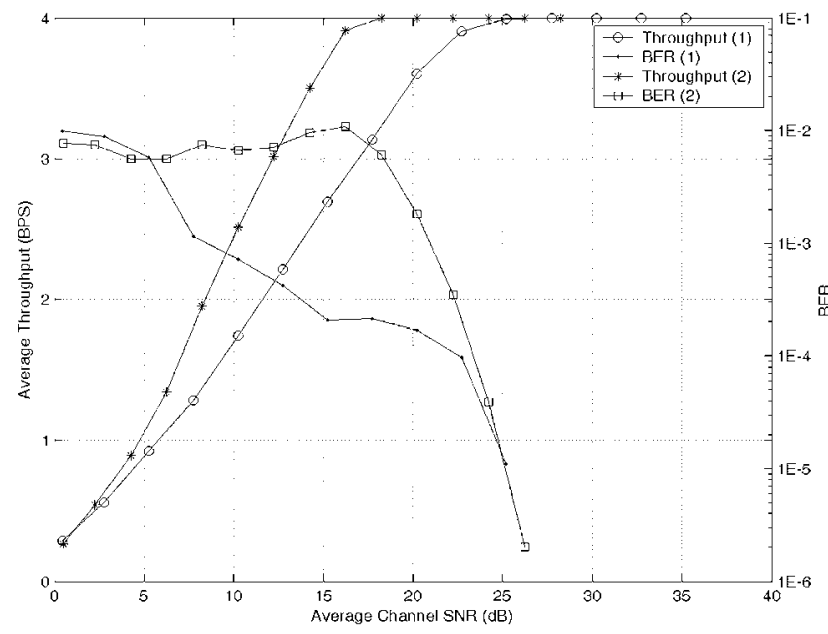

Fig. 4 Average throughput and BER of the adaptive OFDM system when the modulation modes are picked out considering certain switching levels (system '1') and the improvement when we try to maximise (7) with the constraint of (6) never overcome the target BER (system '2')

a fixed modulation mode in all subcarriers, the maximum achievable data rate is $208 \mathrm{Mbits} / \mathrm{s}$ using 16-QAM (four bits by subcarrier symbol, 52 subcarriers).

Fig. 4 shows the average throughput in bits per symbol (BPS) and BER obtained for different average channel SNR values using a four-mode ('no transmission', BPSK, QPSK and 16-QAM) adaptive QAM scheme on a subcarrier-by-subcarrier basis. It is shown to notice that the average throughput for subcarrier symbol (BPS) during the receiver movement regardless of whether individual each subcarrier conveys $1,2,4$ or none data bit. The imposed BER threshold value was $10^{-2}$ and 10 TS were used to characterise the channel response. Using 10 $\mathrm{TS}$, the channel response estimation is very close to the actual channel response and the system performance is practically indistinguishable from the ideal case where a perfect knowledge of the channel response is considered [15]. For this reason, TS $=10$ have been used to estimate the channel response in all simulations. In the results shown in Fig. 4, two cases have been considered: a first system pick out the modulation modes that ensures a certain BER threshold value is not overcome by comparing with switching levels [15]. A second improved system tries to maximise the total throughput given by (7), while maintaining the average error probability given by (6) below the BER threshold. The selection criterion of the 'best subcarrier' is given by (8). We can observe that the new second system have a near-constant BER up to an average channel SNR of $18 \mathrm{~dB}$ and this is close, but below the target BER. Beyond $18 \mathrm{~dB}$, the BER drops dramatically because maximum achievable throughput (four BPS) is attained and the average SNR continues to increasing. Finally, we can observe a significant throughput increase with respect to the previous system, indicated as system ' 1 ' in Fig. 4. As it can be seen, the system ' 1 ' presents a BER that is always rather below the imposed threshold $\left(10^{-2}\right)$ because this system only tries to maintain the instantaneous BER below the imposed threshold for every subcarrier. Thus, a small amount of SNR is wasted in each subcarrier that transmits data. The wasted SNR is greater when the number of subcarriers that convey data is larger. In Fig. 4, we can observe that the BER curves separation is bigger for larger average channel SNR values. When the average channel SNR increases, the number of

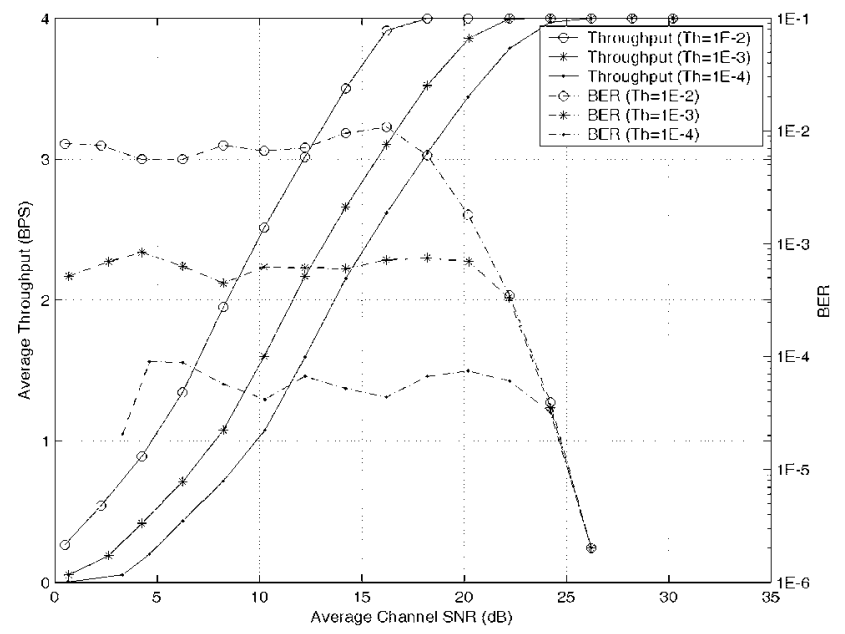

Fig. 5 Performance of the new adaptive scheme for different values of target BER $\left(T h=10^{-2}, 10^{-3}\right.$ and $\left.10^{-4}\right)$

subcarriers in system ' 1 ' that participate in the data transmission is larger, and therefore the amount of wasted SNR augments. Accordingly, the throughput improvement of system ' 2 ' experiments a greater enhancement when the average channel SNR increases. The good performance of this new proposed scheme can also be observed when other target BER values are imposed, as it is shown in Fig. 5. Recently, a near-constant BER and close to the imposed threshold is observed across a wide range of values, while the maximum achievable throughput is not attained. The system performance can be compared with a fixed-mode system that uses the same modulation mode in every subcarrier. In Fig. 6, we can observe that SNR gains between 2.5 and $5 \mathrm{~dB}$ are obtained with the adaptive scheme. Also, the adaptive scheme, in contrast to the previous system presented in [15], reaches the maximum achievable throughput at SNR values very close to those of the fixed-mode system. Finally, Fig. 7 shows the performance of the actual adaptive system (system ' 1 ') compared with that of an ideal system that presents a perfect knowledge of the channel response and the transmitted symbols (system '2'). The good agreement between the results related to both systems indicates a near optimum performance of the new proposed scheme.

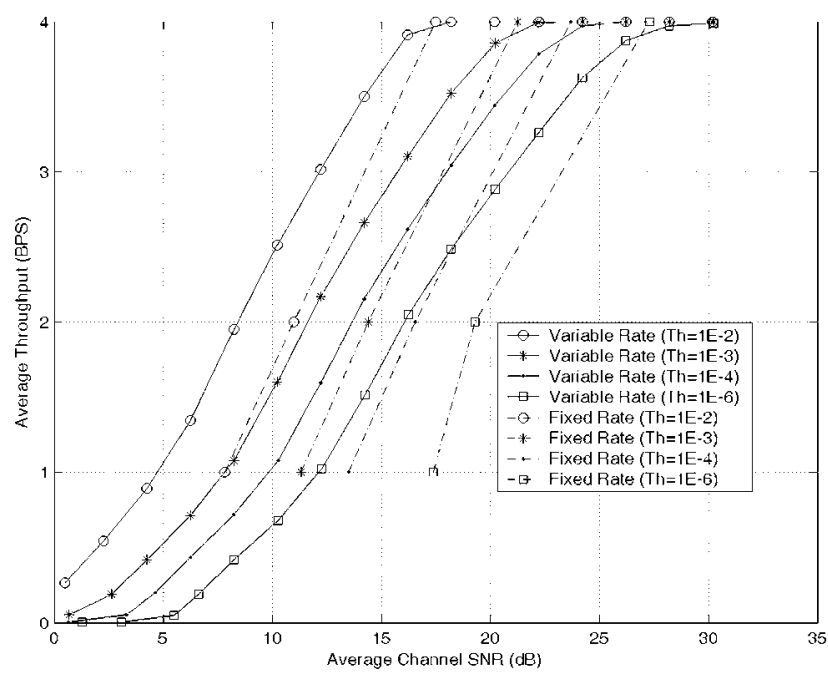

Fig. 6 Comparison of the average throughput of the adaptive scheme and the fixed-mode scheme 


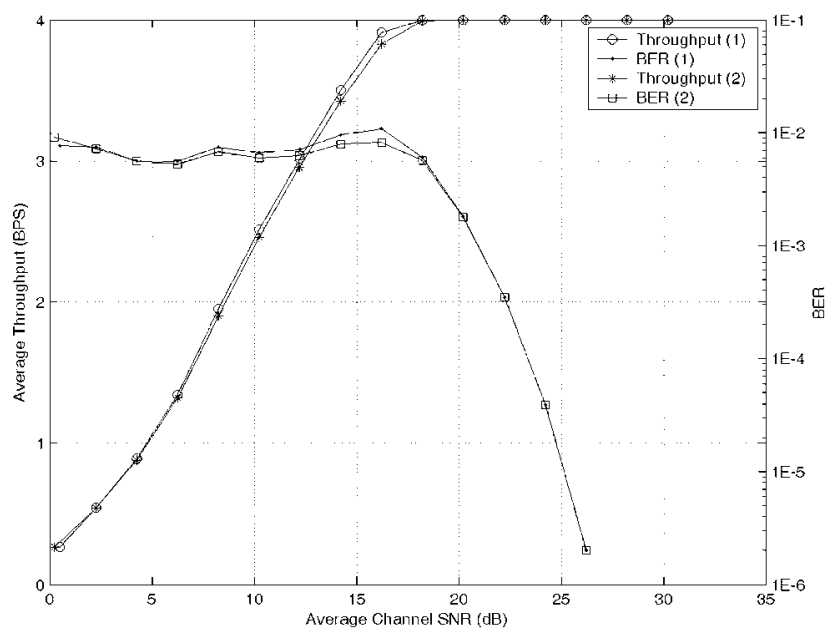

Fig. 7 Average BER of the adaptive QAM scheme for a target $B E R$ of $10^{-2}$ (system ' 1 ') and the results when an ideal situation of perfect knowledge of the channel response and the transmitted symbols is considered (system '2')

\section{Conclusions}

We have proposed an adaptive OFDM system for optical wireless transmission, which is able to withstand multipath dispersion and is more bandwidth efficient than previously presented systems. The OFDM technique allows a great adaptation to the exigent IR channel characteristics, maximising the use of the available bandwidth. The adaptive QAM system described here is able to outperform system throughput over noisy wireless optical channels with respect to fixed-mode OFDM schemes and other previous adaptive systems. The results also show that the new proposed scheme presents a near optimum performance despite its simplicity.

\section{Acknowledgments}

This work has been funded in part by the Spanish Research Adminitration (TIC2003-07005).

\section{References}

1 Kahn, J.M., and Barry, J.R.: 'Wireless infrared communication', Proc. IEEE, 1997, 85, (2), pp. 265-298

2 Marsh, G.W., and Kahn, J.M.: 'Performance evaluation of experimental $50-\mathrm{Mb} / \mathrm{s}$ diffuse infrared wireless link using on-off keying with decision-feedback equalization', IEEE Trans. Commun., 1996, 44, (11), pp. 1496-1504

3 Audeh, M.D., Kahn, J.M., and Barry, J.R.: 'Decision-feedback equalization of pulse-position modulation on measured nondirected indoor infrared channels', IEEE Trans. Commun., 1999, 47, (4), pp. $500-503$

4 Wong, K.K., O'Farrell, T., and Kiatweerasakul, M.: 'Infrared wireless communications using spread spectrum techniques', IEE Proc. Optoelectron., 2000, 147, (4), pp. 308-314

5 Delgado, F., Pérez-Jiménez, R., Rabadán, J., Bacallado, M.A., and López-Hernández, F.J.: 'Experimental characterization of a lowcost fast frequency-hopping spread-spectrum system for wireless in-house optical communications', IEEE Trans. Consum. Electron., 2002, 48, (1), pp. 10-16

6 Carruthers, J.B., and Kahn, J.M.: 'Multiple-subcarrier modulation for nondirected wireless infrared communication', IEEE J. Sel. Areas Commun., 1996, 14, (3), pp. 538-546

7 Kahn, J.M., Krause, W.J., and Carruthers, J.B.: 'Experimental characterization of nondirected indoor infrared channels', IEEE Trans. Commun., 1995, 43, (2-4), pp. 1613-1623

8 Kalet, I.: 'The multitone channel', IEEE Trans. Commun., 1989, 37, (2), pp. $119-124$

9 Hanzo, L., Münster, M., Choi, B.J., and Keller, T.: 'OFDM and MCCDMA for broadband multi-user communications, WLANs and broadcasting' (John Wiley \& Sons, West Sussex, England, 2003)

10 López-Hernández, F.J., Pérez-Jiménez, R., and Santamaría, A.: 'Raytracing algorithms for fast calculation of the channel impulse response on diffuse IR-wireless indoor channels', Opt. Eng., 2000, 39, (10), pp. $1510-1512$

11 Rodríguez, S., Pérez-Jiménez, R., López-Hernádez, F.J., González, O., and Ayala, A.: 'Reflection model for calculation of the impulse response on IR-wireless indoor channels using ray-tracing algorithm', Microw. Opt. Technol. Lett., 2002, 32, (4), pp. 296-300

12 González, O., Rodríguez, S., Pérez-Jiménez, R., Mendoza, B.R. and Ayala, A.: 'Error analysis of the simulated impulse response on indoor wireless optical channels using a Monte Carlo based ray tracing algorithm', IEEE Trans. Commun., 2005, 53, (1), pp. 124-130

13 Lomba, C.R., Valadas, R.T., and Oliveira-Duarte, A.M.: 'Experimental characterisation and modelling of the reflection of infrared signals on indoor surfaces', IEE Proc-Optoelectron., 1998, 145, (3), pp. 191-197

14 Webb, W.T., and Steele, R.: 'Variable rate QAM for mobile radio', IEEE Trans. Commun., 1995, 43, (7), pp. 2223-2230

15 González, O., Pérez-Jiménez, R., Rodríguez, S., Rabadán, J., and Ayala, A.: 'OFDM over the indoor wireless optical channel', IEE Proc., Optoelectron., 2005, 152, (4), pp. 199-204 